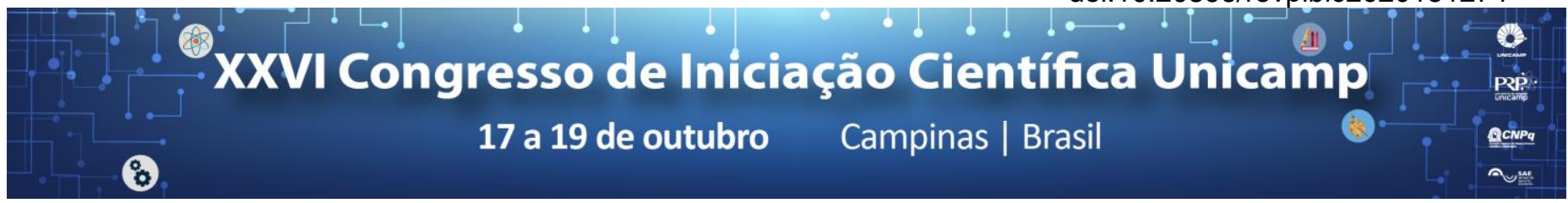

\title{
Development of nanostructured lipid carriers containing the antimicrobial ciprofloxacin for topical application
}

\author{
Talita C. Mendonça*, Simone R. Castro, Marcelo Lancellotti, Lígia N. M. Ribeiro, Eneida de Paula
}

\begin{abstract}
Ciprofloxacin (CIP), a broad spectrum antibacterial agent from the fluoroquinolone family, blocks the activity of the bacterial DNA gyrase. CIP has a limited aqueous solubility and fast clearance rate. Nanostructured lipid carriers (NLC) are drug delivery carriers very appropriate to upload hydrophobic drugs, improving their bioavailability, stability and potency. This work describes the development of NLC containing CIP, their physicochemical characterization and antimicrobial activity, in vitro. NLC particles $(215-355 \mathrm{~nm}$ ) of low size polidispersion (PDI 0.08-0.31) and negative zeta potentials (|20.7-38.7| $\mathrm{mV}$ ) were prepared; scanning electron microscopy revealed their spherical shapes and regular contour. The particles remained stable during 365 days of storage at $25^{\circ} \mathrm{C}$. The encapsulation efficiency of CIP ranged from $72.5-95.2 \%$, with a sustained release profile $(48 \mathrm{~h})$. Preliminary antimicrobial susceptibility tests revealed a $30 \%$ increase in CIP action, when encapsulated in NLC. These proof-of-concept results show the great pharmaceutical and technological potential of the developed nanoformulations. After further tests we believe that NLC-CIP can come to be a novel pharmaceutical form to potentiate the antibiotic effect, reducing its dosage and undesirable side effects.
\end{abstract}

\section{Key words:}

Ciprofloxacin, nanostructured lipid carriers, drug delivery

\section{Introduction}

Ciprofloxacin (CIP) is a fluoroquinolones antibacterial, agent that inhibits the activity of the bacterial DNA gyrase ${ }^{1}$. Lipid-based drug delivery systems such as nanostructured lipid carriers (NLCs) have been shown usefull for the encapsulation of hydrophobic drugs ${ }^{2}$. Therefore, the objective of this work was to encapsulate CIP in NLC, to characterize the formulation and later to evaluate its in vitro antimicrobial activity.

\section{Results and Discussion}

NLCs were prepared by the emulsification-ultrasound method, from a blend of solid and liquid lipids, stabilized by a polymeric surfactant plus $0.3 \%$ CIP. Twelve opaque, homogeneous and líquid formulations were obtained. Dynamic light scaterring analysis revealed particles sizes of: $215.8 \pm 1.55-355.5 \pm 4.79 \mathrm{~nm}$, polidispersity of $0.08 \pm$ $0.033-0.31 \pm 0.017$ and zeta potentials of $-20.7 \pm 0.5--$ $38.7 \pm 1.67 \mathrm{mV}$ (Fig. 1). Scanning electron microscopy confirmed the nanometric size of the NLC, of spherical shape andregular contour.
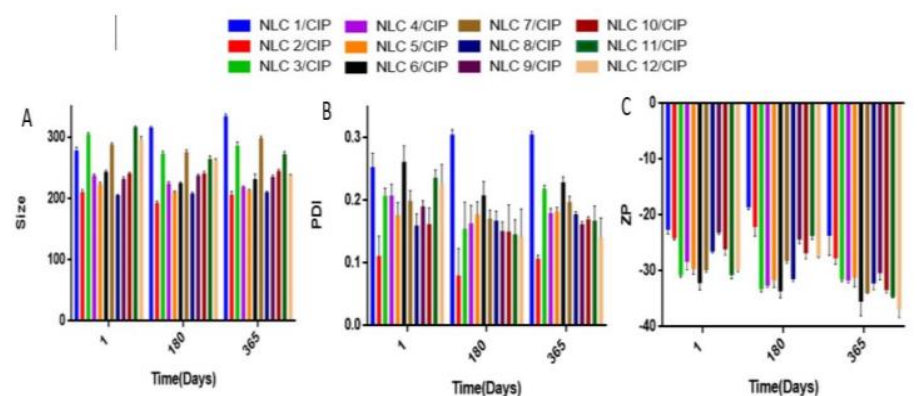

Figure 1: Mean diameter $(A)$, polydispersity index $(B)$ and Zeta potentials (C) of CIP-containing NLC stored for 365 days at $25^{\circ} \mathrm{C}$.

The encapsulation efficiency (\% EE) of CIP in the NLC was high ( 72.5 to $95.2 \%$ ) (Table 1 ), as determined by high performance liquid chromatography (HPLC).
Table 1: Percentage of CIP encapsulation in NLC.

\begin{tabular}{|l|c|l|l|}
\hline Formulation & $\%$ EE & \multicolumn{1}{l|}{ Formulation } & $\%$ EE \\
\hline NLC1 /CIP & 92.25 & NLC7 /CIP & 89.67 \\
\hline NLC2 /CIP & 94.82 & NLC8 /CIP & 72.54 \\
\hline NLC3 /CIP & 94.19 & NLC9 /CIP & 91.78 \\
\hline NLC4 /CIP & 73.47 & NLC10 /CIP & 95.22 \\
\hline NLC5 /CIP & 79.40 & NLC11 /CIP & 94.59 \\
\hline NLC6 /CIP & 92.30 & NLC12 /CIP & 88.87 \\
\hline
\end{tabular}

In vitro release assays revealed that encapsulation into NLC promoted the sustained release of CIP, for more than 48h. Finally, antimicrobial susceptibility tests were performed over 10 gram-positive and gram-negative bacterial strains, including the antibiotic-resistant strains: Staphylococcus aureus, Staphylococcus epidermidis, Pseudomonas aeruginosa, Klebsiella pneumoniae and Escherichia coli. Encapsulation into the NLC potentiated the antibacterial activity of CIP (10 - 30\%) in relation to the control (free CIP), being effective also against the antibiotics-resistant bacteria.

\section{Conclusions}

NLC-CIP formulations showed desirable physicochemical features, good stability, sustained release profile and potentiated the antimicrobial activity of ciprofloxacin. We conclude that these nanostructuted lipid-formulations can be seen as promising new pharmaceutical forms to potentiate the effect of antibiotics.

\section{Acknowledgement}

The authors thank the support from CNPq (T.C.M., E.P fellowships) and FAPESP (\# 2014/14457-5).

\footnotetext{
1 YALVAC, I. S.; BASCI, N. E.; BOZKURT, A.; DUMAN, S. Penetration of topically applied ciprofloxacin and ofloxacin into the aqueous humor and vitreous. J. Cataract Refract Surg, 29, 487-491, 2003.
}

2 BELOQUI, A. et al. Nanostructured lipid carriers: Promising drug delivery systems for future clinics. Nanomedicine: Nanotechnology, Biology, and Medicine, v. 12, n. 1, p. 143-161, 2016. 